\title{
Balanced Directional Coupler Structure with Insensitive Isolation for Load Impedance
}

\author{
Won-Gyu Lim ${ }^{\# 1}$, Seo-Young Park ${ }^{\# 2}$, Sang-Yun Lee ${ }^{\# 3}$, Moon-Que Lee ${ }^{* 4}$, Jong-Won Yu ${ }^{\# 5}$ \\ ${ }^{\#}$ School of Electrical Engineering and Computer Science, Korea Advanced Institute of Science and Technology \\ 373-1 Guseong-Dong, Yuseong-Gu, Daejeon, 305-701, Korea \\ *School of ECE, University of Seoul, Seoul, 130-743, Korea \\ ${ }^{1}$ limwongyu@kaist.ac.kr \\ purplemist@kaist.ac.kr \\ gjsqud2007@kaist.ac.kr \\ ${ }^{4}$ mqlee@uos.ac.kr \\ ${ }^{5}$ drjwyu@ee.kaist.ac.kr
}

\begin{abstract}
In this paper, a balanced directional coupler structure with lumped elements is presented as a new method to solve Txto-Rx leakage problem. An analysis for the characteristics of the balanced directional coupler is performed to introduce that the proposed structure is insensitive for varying load impedance. The balanced directional coupler structure consists of two Wilkinson power dividers and two contra-directional couplers implemented with lumped elements. The validity of the proposed structure is confirmed by comparisons with the measured results obtained from a single contra-directional coupler. The fabricated balanced directional coupler has $58 \mathrm{~dB}$ of isolation for matched load impedance and less than $45 \mathrm{~dB}$ for mismatched load impedance at $910 \mathrm{MHz}$, the center frequency of UHF RFID.
\end{abstract}

Index terms -Directional coupler, Tx leakage, Front-end, UHF RFID, Isolation.

\section{INTRODUCTION}

In microwave circuits, components such as circulators and directional couplers have been used to guarantee the isolation between transmitter and receiver [1], [2]. Because the reading range should be as long as a few meters in the UHF RFID system, the isolation between a transmitter and a receiver which operates simultaneously at the same frequency has always been an important issue. However, conventional components provide insufficient isolation around $25 \mathrm{~dB}$ and this feature is also sensitive for a mismatched load, especially antenna. Hence, diverse methods have been studied [3]-[4].

In this paper, we propose the balanced circulator with high isolation for varying load impedance, as shown in Fig. 2. The basic principle of the balanced structure is to cancel the direct coupler leakage signal (denoted as $I$ in Fig. 1) by adding $180^{\circ}$ delayed (anti-phase) signal to $\mathrm{Rx}$ port through the compensation circuit (shaded region of Fig. 2). Therefore, we approved this principle through theoretical and experimental verification for variant possibilities of load impedance change. In addition, the proposed structure can take advantages of the low-cost and light-weight characteristics from implementing with lumped elements.

For many practical applications, the balanced antennas, such as dipole antenna, quadrifilar spiral antenna, and

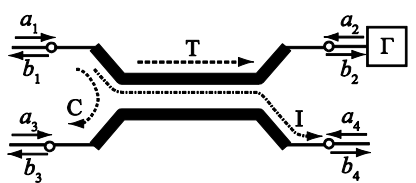

Fig. 1. Conventional contra-directional coupler with mismatched load.

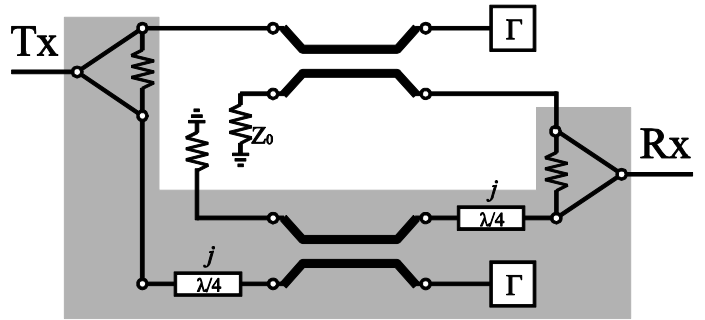

Fig. 2. Proposed balanced directional coupler structure.

quadrifilar helix antenna [4], can be connected with the proposed balanced circulator.

\section{RELEVANT THEORY}

\section{A. Isolation}

In Fig. 1, the single contra-directional coupler is connected to the mismatched load. The scattering matrix of this conventional coupler is represented in (1).

$$
\left[\begin{array}{l}
b_{1} \\
b_{2} \\
b_{3} \\
b_{4}
\end{array}\right]=\left[\begin{array}{cccc}
0 & \mathrm{~T} & \mathrm{C} & \mathrm{I} \\
\mathrm{T} & 0 & \mathrm{I} & \mathrm{C} \\
\mathrm{C} & \mathrm{I} & 0 & \mathrm{~T} \\
\mathrm{I} & \mathrm{C} & \mathrm{T} & 0
\end{array}\right]\left[\begin{array}{l}
a_{1} \\
a_{2} \\
a_{3} \\
a_{4}
\end{array}\right]
$$

, where $a_{\mathrm{n}}$ and $b_{\mathrm{n}}$ represents an incident wave and a reflected wave at the $n$-th port, respectively as shown in the above figure. The capital letters T, I, C are used for convenience, which indicate the factors of through, isolation and coupled.

If we assume that the reader antenna has mismatched load and the port 3 is connected to a $50 \Omega$ terminal, the total Tx leakage of a single directional coupler is 
Tx

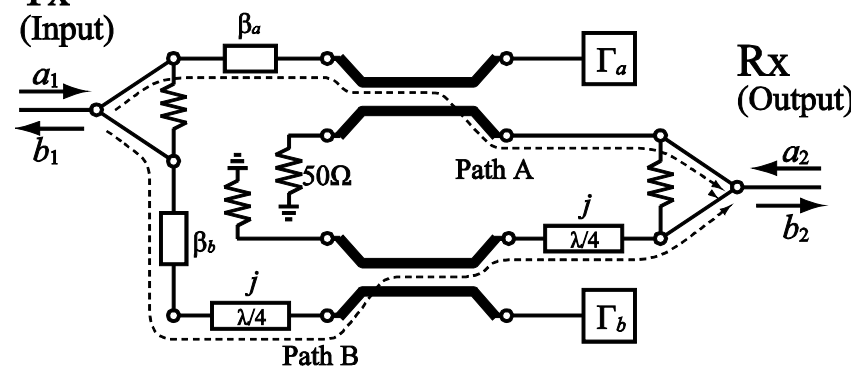

Fig. 3. Equivalent circuits of the balanced coupler with mismatched load and path error.

$$
b_{4} / b_{1}=I+T \cdot C \cdot \Gamma
$$

, which implies that the isolation characteristic is affected by both the isolation of directional coupler and the reflection coefficient of the reader antenna.

Fig. 3 shows the equivalent circuits of the balanced directional coupler, which have two directional couplers, two power dividers and two load connections. $\beta_{a}$ and $\beta_{b}$ indicate the amplitude and phase errors of the compensated circuits in path A and B, respectively.

For the balanced directional coupler with mismatched load, the scattering matrix is given in (5).

Therefore, the Tx leakage of the balanced directional coupler with the mismatched load is,

$$
\begin{aligned}
& \frac{b_{2}}{a_{1}}=\frac{1}{2}\left(\mathrm{TC}\left(\beta_{a} \Gamma_{a}-\beta_{b} \Gamma_{b}\right)+\mathrm{I}\left(\beta_{a}-\beta_{b}\right)\right), \\
& \frac{b_{2}}{a_{1}}=\frac{1}{2} \mathrm{~T} \cdot \mathrm{C} \cdot\left(\Gamma_{a}-\Gamma_{b}\right), \text { for the ideal case }
\end{aligned}
$$

, where $\Gamma_{a}$ and $\Gamma_{b}$ are reflection coefficients of antenna ports. In above equation, the Tx leakage can be considered for two cases. The first case is that the path $\mathrm{A}$ and $\mathrm{B}$ are not equal and the antennas are balanced $\left(\Gamma_{a}=\Gamma_{b}\right)$. Second, the antennas are unbalanced and the path $\mathrm{A}$ and $\mathrm{B}$ are equal $\left(\beta_{a}=\beta_{b}\right)$. The Tx leakages for above-mentioned cases are given in (6), respectively.

$$
\begin{array}{ll}
\frac{b_{2}}{a_{1}}=\frac{1}{2} \mathrm{~T} \cdot \mathrm{C} \cdot \beta \cdot\left(\Gamma_{a}-\Gamma_{b}\right), & \text { for } \beta_{a}=\beta_{b}=\beta \\
\frac{b_{2}}{a_{1}}=\frac{1}{2}(\mathrm{~T} \cdot \mathrm{C} \cdot \Gamma+\mathrm{I}) \cdot\left(\beta_{a}-\beta_{b}\right), & \text { for } \Gamma_{a}=\Gamma_{b}=\Gamma
\end{array}
$$

From the above equations, it is noted that high isolation can be achieved whenever one of these two factors $(\beta$ and $\Gamma)$ is in balance although the other one is somehow unbalanced. The calculated isolation characteristics are shown in Fig. 4 for two cases.

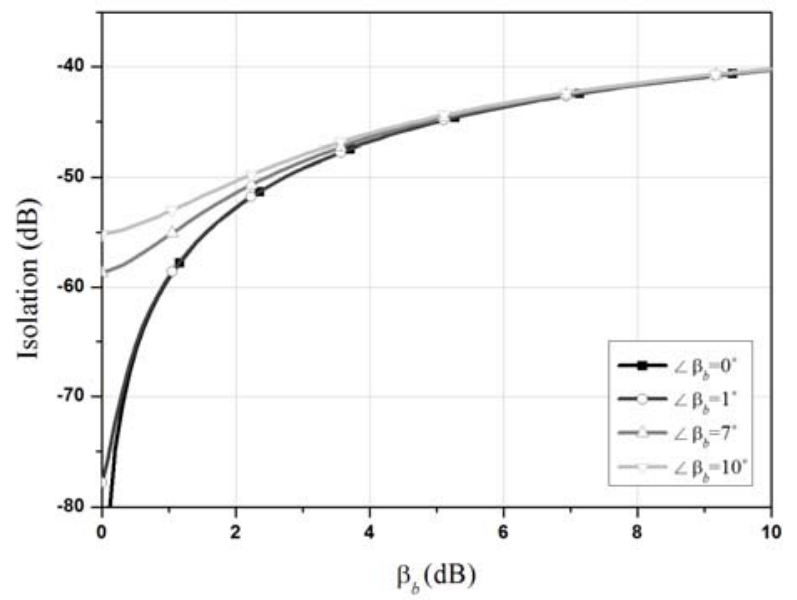

(a)

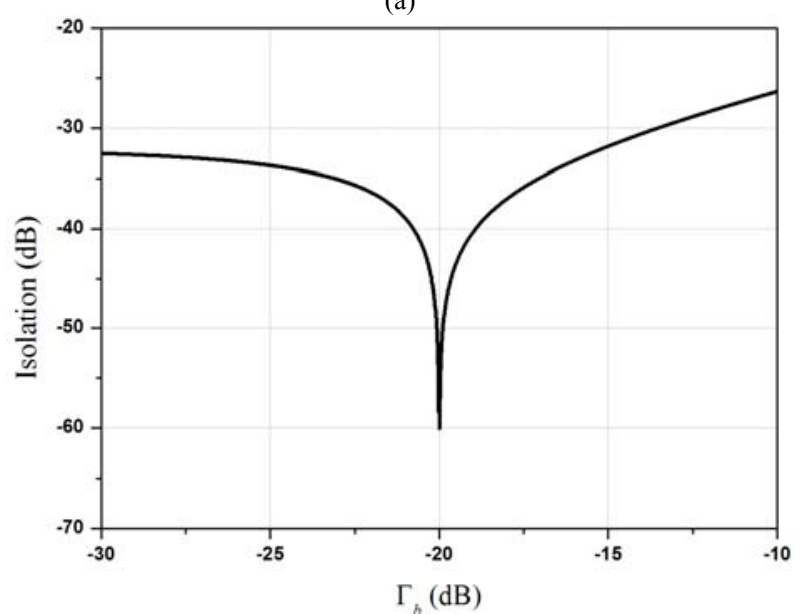

(b)

Fig. 4. Calculated isolation characteristics for the proposed structure. (a) Unbalanced path error with balanced antenna. (b) Balanced path error with unbalanced antenna.

In case (a), the error from the path $\mathrm{B}\left(\beta_{b}\right)$ varies in magnitude for given phase errors while the antennas are balanced $\left(\Gamma_{a}=\Gamma_{b}=-20 \mathrm{~dB}\right)$. In case (b), mismatched load impedance are applied on the proposed structure with the balanced path $\left(\beta_{a}=\beta_{b}=1 \angle 0^{\circ}\right)$. It is noted that the proposed structure shows isolation characteristics which are robust for varying load impedance and path loss and relatively better than that of a single directional coupler.

\section{MEASUREMENT RESULT}

To implement the balanced directional coupler structure using lumped elements for UHF RFID system, the design of Wilkinson power dividers and $\pi$-type $12 \mathrm{~dB}$ contra-directional couplers, the dominant composers of the proposed structure is preceded, based on conventional method as in Fig. 5 [5], [6].

$$
\left[\begin{array}{l}
b_{1} \\
b_{2}
\end{array}\right]=\frac{1}{2}\left[\begin{array}{cc}
\mathrm{T}^{2}\left(\beta_{a}^{2} \Gamma_{a}-\beta_{b}^{2} \Gamma_{b}\right) & \mathrm{TC}\left(\beta_{a} \Gamma_{a}-\beta_{b} \Gamma_{b}\right)+\mathrm{I}\left(\beta_{a}-\beta_{b}\right) \\
\mathrm{TC}\left(\beta_{a} \Gamma_{a}-\beta_{b} \Gamma_{b}\right)+\mathrm{I}\left(\beta_{a}-\beta_{b}\right) & \mathrm{C}^{2}\left(\Gamma_{a}-\Gamma_{b}\right)
\end{array}\right]\left[\begin{array}{l}
a_{1} \\
a_{2}
\end{array}\right]
$$




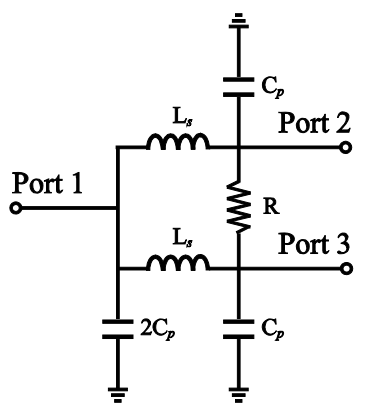

(a)

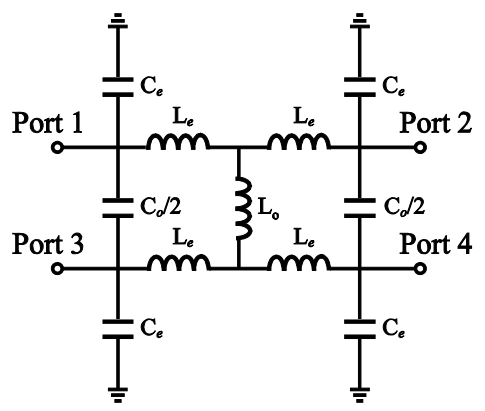

(b)
Fig. 5. Schematics of (a) Wilkinson power divider and (b) contra-directional coupler.

$$
\begin{aligned}
C_{e} & =\frac{Y_{0 e}}{\omega} \tan \frac{\theta}{2}, L_{e}=\frac{Z_{0 e}}{2 \omega} \sin \theta \\
C_{o} & =-\frac{Y_{0 o}}{\omega} \cot \frac{\theta}{2}+\frac{1}{\omega^{2} L_{e}}-C_{e} \\
L_{o} & =\frac{1}{\omega^{2}\left(C_{e}+C_{o}\right)-\omega Y_{0 o} \tan \frac{\theta}{2}}
\end{aligned}
$$

Using the above equations, the detailed component values can be calculated: $\mathrm{C}_{p}=2 \mathrm{pF}, \mathrm{L}_{s}=12.4 \mathrm{nH}$ and $\mathrm{R}=100 \Omega$ for the Wilkinson power divider, $\mathrm{C}_{e}=2.7 \mathrm{pF}, \mathrm{L}_{e}=2.7 \mathrm{nH}, \mathrm{C}_{o}=3.6 \mathrm{pF}$ and $\mathrm{L}_{o}=3.3 \mathrm{nH}$ for the $12 \mathrm{~dB}$ contra-directional coupler, operating at UHF RFID frequency. The fabricated Wilkinson power divider on FR4 substrate $\left(\varepsilon_{\mathrm{r}}=4.6\right)$ with $0.6 \mathrm{~mm}$ thickness showed $-3.4 \mathrm{~dB}$ for both $\mathrm{S} 21$ and S31 at $910 \mathrm{MHz}$. Also, the implemented $12 \mathrm{~dB}$ contra-directional coupler had about $-0.8 \mathrm{~dB},-12.5 \mathrm{~dB}$ and $-38 \mathrm{~dB}$ at $910 \mathrm{MHz}$ for $\mathrm{S}$ parameters of the through, coupled and isolation port, respectively. The balanced directional coupler circuits are also fabricated on the FR4 $\left(\varepsilon_{\mathrm{r}}=4.6\right)$ substrate with $0.6-\mathrm{mm}$ thickness.

The measured results of the isolation characteristics for the single contra-directional coupler in varying load impedance condition $(25 \Omega, 50 \Omega, 75 \Omega$ and $100 \Omega$ ) are shown in Fig. 6 . In perfectly matched case, about $38.9 \mathrm{~dB}$ of isolation is obtained at $910 \mathrm{MHz}$ (black solid line with filled triangles). As the load impedance varies, the isolation is measured from $22.1 \mathrm{~dB}$ to $25.4 \mathrm{~dB}$ (dark gray and light gray solid lines). The isolation characteristic of the proposed structure at the UHF RFID frequency band is shown in Fig. 7.

When there is no reflection from the antenna load, the isolation feature is measured about $58.1 \mathrm{~dB}$ at $910 \mathrm{MHz}$ (black solid line with filled triangles). Also, the proposed structure provided about $45.4 \mathrm{~dB}$ of isolation for $100 \Omega$ of mismatched load (light gray solid line with inverted triangles), which is about $20 \mathrm{~dB}$ better than the results of the conventional directional coupler.

In this structure, the Tx power are about $-4.3 \mathrm{~dB}$ at two antenna ports. If we employ two-port balanced antenna, the Tx power will experience about $-1.3 \mathrm{~dB}$ of propagation loss.

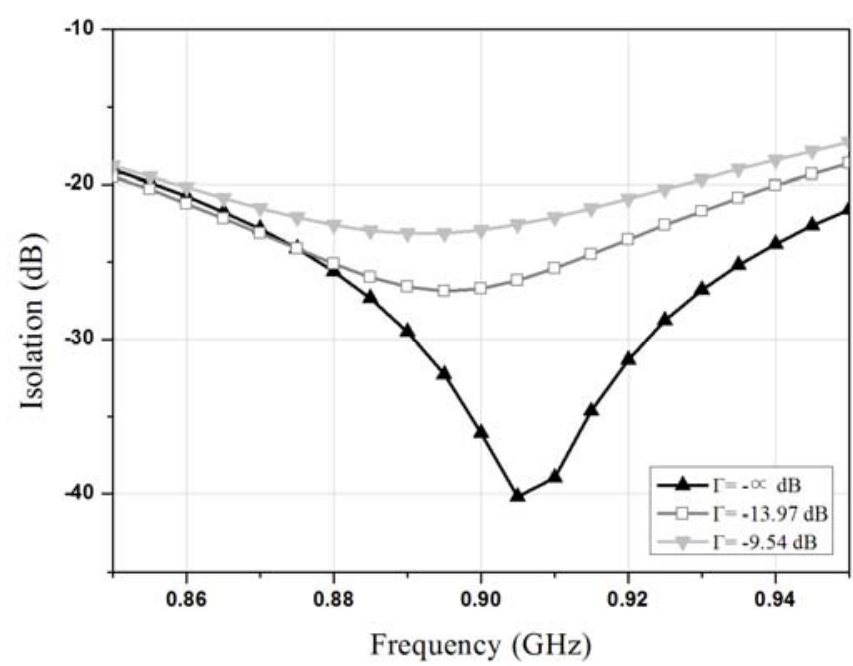

Fig. 6. Measured isolation characteristics of conventional directional coupler with mismatched load.

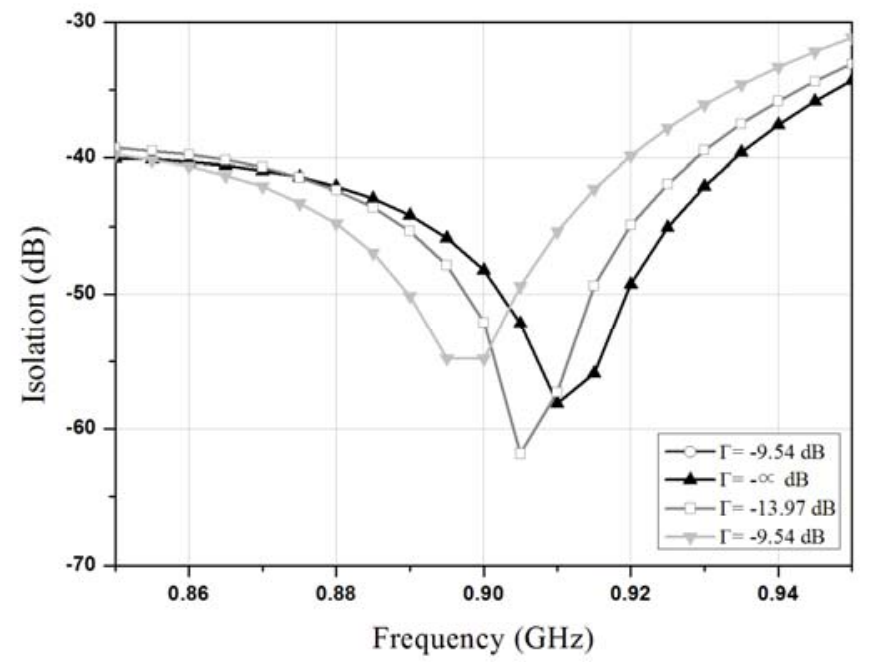

Fig. 7. Measured isolation characteristics of balanced directional coupler with mismatched load.

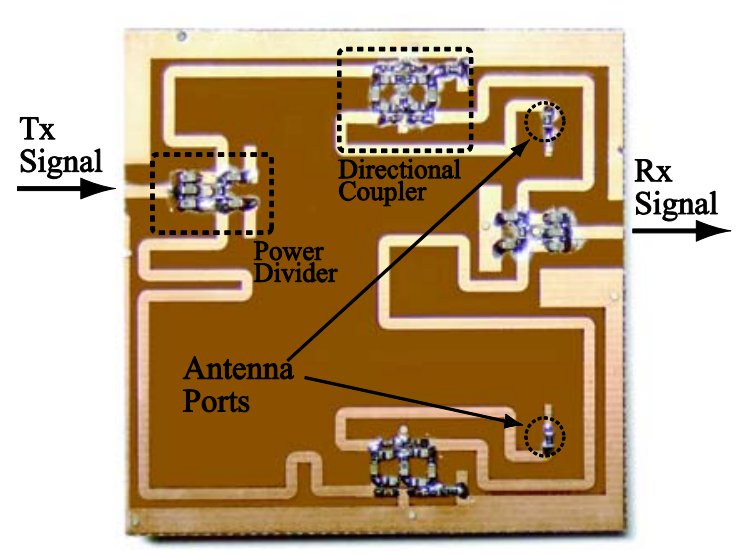

Fig. 8. Fabricated prototype of the balanced directional coupler. 


\section{CONCLUSIONS}

In this paper, we proposed a balanced directional coupler structure with high isolation and load-insensitive characteristics. This improved isolation characteristic is achieved by employing two power divider and two contradirectional coupler to cancel the Tx leakage signal. These features can contribute for the performance of RFID reader.

\section{ACKNOWLEDGEMENT}

This research was partially supported by the Brain Korea 21 Project, the School of Information Technology, KAIST in 2008 and the IT R\&D program of MKE/IITA [2007-F-043-02, Study on Diagnosis and Protection Technology based on EM].

\section{REFERENCES}

[1] Katariina Penttilä, Lauri Sydänheimo and Markku Kivikoski, "Implementation of Tx/Rx isolation in an RFID reader," Int. J. Radio Frequency Identification Technology and Applications, Vol. 1, No. 1, 2006.

[2] Won-Gyu Lim and Jong-Won Yu, "Balanced Circulator Structure with Enhanced Isolation Characteristics," IEEE Antennas and Wireless Propagation Lett., accepted for publication.

[3] Wan-Kyu Kim, Moon-Que Lee, Jin-Hyun Kim, Hyung-sun Lim, JongWon Yu, Byung-Jun Jang and Jun-Seok Park, "A passive circulator with high isolation using a directional coupler for RFID," IEEE 2006 MTT-S Int. Microwave Symp. Dig.,pp.196-199, Jun. 2006.

[4] Peng Bai, Yingzeng Yin and Xi Yang, "A novel Rx-Tx front-ends for passive RFID reader with high isolation," IEEE 2007 Int. Symp. on MAPE, pp.332-335, 2007.

[5] W. I. Son, W. G. Lim, M. Q. Lee, S. B. Min, and J. W. Yu, "Printed Square Quadrifilar Spiral Antenna for UHF RFID Reader," IEEE AP-S International Symposium, pp. 305-308, June 10-15, 2007.

[6] D. Pozar, Microwave Engineering, 3rd edition, John Wiley \& Sons, Inc., 2005.

[7] R. Mongia, I. Bahel, and P. Bhartia, $R F$ and microwave coupled line circuits, Artech House, Boston, 1999, pp.181 - 193. 